\title{
Alterations in liver sinusoidal endothelium in a baboon model of type 1 diabetes
}

\author{
H. A. Jamieson • V. C. Cogger • S. M. Twigg • \\ S. V. McLennan - A. Warren • R. Cheluvappa • \\ S. N. Hilmer • R. Fraser • R. de Cabo • D. G. Le Couteur
}

Received: 6 December 2006 / Accepted: 28 May 2007 / Published online: 29 June 2007

(C) Springer-Verlag 2007

\begin{abstract}
Aims/hypothesis Diabetes mellitus is associated with extensive vascular pathology, yet little is known about its longterm effects on liver sinusoidal endothelial cells (LSECs).
\end{abstract}

H. A. Jamieson • V. C. Cogger • A. Warren · R. Cheluvappa •

S. N. Hilmer $\cdot$ D. G. Le Couteur

Centre for Education and Research on Ageing, Concord Hospital,

Sydney, NSW, Australia

H. A. Jamieson $(\bowtie) \cdot$ V. C. Cogger $\cdot A$. Warren · R. Cheluvappa

D. G. Le Couteur

ANZAC Research Institute,

Concord RG Hospital and University of Sydney,

Concord, NSW 2139, Australia

e-mail: hjamieson@med.usyd.edu.au

H. A. Jamieson $\cdot$ R. de Cabo

Laboratory of Experimental Gerontology,

National Institute on Aging, National Institutes of Health,

Baltimore, MD, USA

\section{S. M. Twigg $\cdot$ S. V. McLennan}

Department of Endocrinology, Royal Prince Alfred Hospital

and University of Sydney,

Camperdown, NSW, Australia

\section{S. N. Hilmer}

Department of Aged Care, Royal North Shore Hospital,

St Leonards, NSW, Australia

\section{S. N. Hilmer}

Department of Clinical Pharmacology,

Royal North Shore Hospital,

St Leonards, NSW, Australia

\section{R. Fraser}

Department of Pathology, Christchurch School of Medicine,

University of Otago,

Christchurch, New Zealand
Potential diabetic changes in LSECs are important because of the role played by fenestrations in the LSECs in hepatic disposition of lipoproteins.

Materials and methods Surgical liver biopsies for electron microscopy and immunohistochemistry were obtained from baboons with long-standing streptozotocin-induced, insulin-treated diabetes mellitus and compared with those from age-matched control animals.

Results There was an increase in the thickness of LSECs $(170 \pm 17$ vs $123 \pm 10 \mathrm{~nm}, p<0.01)$. Fenestrations in LSECs, as determined by overall porosity, were markedly reduced $(1.4 \pm 0.1 \%$ vs $2.6 \pm 0.2 \%, p<0.01)$. Increased numbers of stellate cells were seen on electron microscopy, and this finding was corroborated by increased smooth muscle actin expression. Diabetes mellitus was also associated with increased endothelial production of von Willebrand factor and caveolin-1.

Conclusions/interpretation Diabetes mellitus in the nonhuman primate is associated with marked changes in LSECs, including a reduction in fenestrations. Such changes provide an additional and novel mechanism for impaired hepatic lipoprotein clearance and post-prandial hyperlipidaemia in diabetes mellitus.

Keywords Diabetes mellitus · Electron microscopy . Fenestrations · Liver sinusoidal endothelial cell $\cdot$ LSEC . Papio hamadryas $\cdot$ Stellate cells

$\begin{array}{ll}\text { Abbreviations } \\ \text { LSECs } & \text { liver sinusoidal endothelial cells } \\ \text { PAS } & \text { periodic acid-Schiff } \\ \text { SMA } & \alpha \text {-smooth muscle actin }\end{array}$




\section{Introduction}

The vascular complications of diabetes mellitus are well established and have major clinical significance [1]. However, little is known about the effects of diabetes mellitus on the hepatic microvascular system. The hepatic microvasculature consists of sinusoids, which are porous, gossamer-like vessels that connect afferent portal triads to exiting central hepatic venules over a distance of about $1 \mathrm{~mm}$. Approximately one billion sinusoids form the rich capillary network of the liver, which permits the vast hepatic blood flow to course slowly and intimately between the cords of hepatocytes $[2,3]$.

Liver sinusoidal endothelial cells (LSECs) are the highly specialised endothelial cells that line the wall of the hepatic sinusoid and separate the sinusoidal blood, which is derived primarily from the portal vein, from hepatocytes. LSECs are perforated with fenestrations, which are pores approximately 50 to $150 \mathrm{~nm}$ in diameter grouped together in clusters known as liver sieve plates. Fenestrations are true discontinuities in the endothelium, lacking either a diaphragm or underlying basal lamina. They facilitate the passage of macromolecules into the extravascular space for subsequent hepatocellular uptake and metabolism. The cytoplasmic extensions of LSECs are extremely thin $(100-200 \mathrm{~nm})$, which also promotes the transport of smaller, diffusible substrates [3]. Of interest in diabetes mellitus is the observation that fenestrations in the LSECs appear to act as conduits for the transfer of some lipoproteins, especially chylomicron remnants, between the blood and hepatocytes [2,4]. In old age, a substantial loss of fenestrations in the LSECs occurs [5-8], which impairs lipoprotein transfer to the hepatocyte [9]. This provides a mechanism for age-related impairment in chylomicron remnant clearance and post-prandial hypertriacylglycerolaemia, thus possibly contributing to enhanced vascular risk among older people [4]. As such, there are potential parallels with dyslipidaemia in patients with diabetes mellitus [10-12].

The paucity of research into the effects of diabetes mellitus on the LSECs probably reflects the considerable risk of liver biopsy in humans. Furthermore, many experimental animal models do not fully reflect the metabolic changes seen in humans with diabetes mellitus $[13,14]$. The Australian National Primate colony has a long established non-human primate colony (Papio hamadryas), in which type 1 diabetes mellitus was induced by streptozotocin administered at an average 2 years of age [14]. Streptozotocin destroys the insulin-secreting beta cells of the pancreas and creates a hypoinsulinaemic, hyperglycaemic state that is similar to type 1 diabetes mellitus [15]. In many animal models, streptozotocin-induced diabetes mellitus has been shown to closely resemble that seen in humans [14] and such models are generally considered to be excellent for the study of type 1 diabetes [16]. The hyperglycaemia in these baboons is partly controlled with once daily insulin injections to avoid weight loss and it has been possible to obtain a degree of control of type 1 diabetes similar to that in less well controlled humans with type 1 diabetes [14]. The diabetic baboons have non-diabetic aged-matched controls. Previous data from the kidney and peripheral nerves of animals from this same baboon colony have led researchers to the conclusion that the structural and functional changes are similar to those seen in human diabetes and different from those seen in diabetic rats $[17,18]$.

In this work, we obtained open liver biopsies from diabetic baboons and age-matched controls in order to study the effects of chronic, insulin-treated type 1 diabetes mellitus on the ultrastructure of the hepatic microvascular. We particularly wanted to determine whether diabetes mellitus influences fenestrations in LSECs because of potential mechanistic implications for diabetic dyslipidaemia.

\section{Materials and methods}

Animals and specimen collection Baboons were from the National Baboon Colony (Sydney, NSW, Australia) and are members of a breeding colony of Papio hamadryas. Streptozotocin $(60 \mathrm{mg} / \mathrm{kg}$, intravenous) had been injected into the baboons at an average of 2 years of age to create a diabetic state [14, 17, 18]. Capillary blood glucose levels are measured every other day in the diabetic baboons and subcutaneous insulin (human regular and ultralente, $\sim 4$ units in total) is administered daily according to the trend of glucose levels and $\mathrm{HbA}_{1 \mathrm{c}}$ levels. The overall target levels are: random plasma glucose $15-30 \mathrm{mmol} / \mathrm{l}$; and $\mathrm{HbA}_{1 \mathrm{c}} 8.0$ $10.0 \%$, reflecting sub-optimally controlled human type 1 diabetes. All animals are housed in family groups and are fed a standard diet. Supervision is by experienced veterinarians. Ethics approval for this procedure was obtained from the Central Sydney Area Health Service Animal Welfare Ethics Committee and the work was conducted in accordance with the Principles of Laboratory Animal Care (NIH Publication 85-23, revised 1985).

Four diabetic baboons aged 10-15 years, with diabetes duration of approximately 10 years, were randomly chosen from the colony. Four non-diabetic control animals were matched with the diabetic baboons based on age and body weight. They were fasted on the night before the procedure. Intramuscular ketamine was used to induce general anaesthesia. Blood was taken $(5 \mathrm{ml})$ for measurement of lipids, urea and electrolytes, glucose, $\mathrm{HbA}_{1 \mathrm{c}}$, full blood count and liver function tests (determined by the NATA accredited Department of Biochemistry Laboratory, Royal Prince 
Alfred Hospital, Australia). A $1 \mathrm{~cm}^{3}$ open liver biopsy was taken by an experienced human and primate surgeon. The baboons were closely observed for $24 \mathrm{~h}$ after the procedure for any signs of complications. All the animals tolerated the procedure well and made full post-operative recovery.

Approximately one-third of the liver biopsy was fixed in $4 \%(w / v)$ phosphate-buffered paraformaldehyde. The remaining liver was perfusion-fixed for electron microscopy as previously published [6]. An aliquot (1-2 ml) of fixative $(2 \%[w / v]$ glutaraldehyde/3\% $[w / v]$ paraformaldehyde in $0.1 \mathrm{~mol} / 1$ sodium cacodylate buffer, $0.1 \mathrm{~mol} /$ 1 sucrose, $2 \mathrm{mmol} / 1 \mathrm{CaCl}_{2}$ ) was injected slowly into the liver sample using a $25 \mathrm{G}$ needle until the tissue hardened.

Scanning electron microscopy Fixed liver tissue was treated with $1 \%(w / v)$ osmium then dehydrated in an ethanol gradient to $100 \%$ and incubated for $10 \mathrm{~min}$ in hexamethyl-disilazane (Sigma, St Louis, MO, USA). Samples were mounted and then splutter-coated with gold. Specimens were visualised using a scanning electron microscope (JSM 6380; Jeol, Sydney, NSW, Australia). Twelve random sinusoids, at $\times 25,000$ magnification, were photographed from each liver. Analysis of fenestrae diameter and frequency was made using a specific software package (Image J; National Institutes of Health, Bethesda, MD, USA).

Transmission electron microscopy Fixed liver tissue was processed and embedded in Spurr's resin. Blocks were sampled at random for light microscopy assessment and two blocks from each liver were selected from those satisfying tissue integrity and fixation quality requirements. Ultra thin sections were taken from each block and stained with uranyl acetate then lead citrate. Then 12 random sinusoids from each liver were viewed at $\times 20,000$ magnification, using a transmission electron microscope (Zeiss 902; Carl Zeiss, Sydney, NSW, Australia). The images were captured on a slow scan CCD camera (Gatan BioScan; Thomson Scientific Instruments, Melbourne, VIC, Australia). Ten measurements of endothelial thickness were made to determine the width of each sinusoid using Image $\mathrm{J}$.

Light microscopy Paraformaldehyde-fixed liver was embedded in paraffin blocks and $5 \mu \mathrm{m}$ sections were taken. Samples were stained with haematoxylin and eosin, Masson's trichrome, reticulin and periodic acid-Schiff (PAS) for light microscopy.

Immunohistochemistry was used to determine the production of caveolin-1, collagen IV and von Willebrand factor. Deparaffinised sections underwent antigen retrieval with proteinase $\mathrm{K}$ (for collagen IV and von Willebrand factor) and pressure cooker (for caveolin-1 and $\alpha$-smooth muscle actin [SMA]). Endogenous peroxidase was blocked with $3 \%(v / v)$ hydrogen peroxide. The primary antibodies, which were applied for $1 \mathrm{~h}$, were goat anti-human collagen IV (Zymed, San Francisco, CA, USA), mouse anti-human von Willebrand factor (Dako, Glostrup, Denmark) rabbit anti-human caveolin-1 (Santa Cruz Biotechnology, Santa Cruz, CA, USA) and mouse anti-human SMA (Sigma, Poole, UK). Sections were then treated with goat antirabbit IgG, poly horseradish peroxidase (Chemicon, Temecula, CA, USA). 3,3'-Diaminobenzidine was used to reveal peroxidase activity. Sections were counterstained with haematoxylin. Staining was rated blindly and independently by three researchers. Masson's trichrome, reticulin caveolin-1, collagen IV and von Willebrand factor staining were rated on a scale between 0 (absent staining), + (light staining) and +++ (heavy staining) for the intensity of staining in the periportal sinusoids, pericentral sinusoids, portal vein and central veins. PAS staining was rated by the percentage of hepatocytes staining positive. SMA was assessed by counting the number of SMA-positive cells per field in four random fields per baboon at $\times 20$ magnification. The researchers then reviewed the slides together and gave consensus ratings for the staining intensity on each slide.

Statistics Results are expressed as means \pm SEM. Comparisons of the blood results and electron microscopy data were performed using either the Student's $t$ test or MannWhitney rank sum test for nonparametric data. Differences were considered significant at $p<0.05$.

\section{Results}

Age, weights and blood results Diabetic and control animals were well matched for body weight and age (Table 1). Fasting blood analysis showed significantly elevated $\mathrm{HbA}_{1 \mathrm{c}}$ and glucose in the diabetic group. Diabetic animals also had microalbuminuria as indicated by analyses of timed urinary excretion rates. Liver function tests were mildly elevated in the diabetic group with significant increases in alkaline phosphatase, gammaglutamyl transferase, alanine transaminase and aspartate transaminase. There were no differences in bilirubin or creatinine. Plasma triacylglycerol levels were increased substantially (approximately seven to eightfold) in the diabetic group.

Scanning electron microscopy The porosity of the LSECs was significantly reduced to nearly one half in the diabetic livers ( $1.4 \pm 0.1$ vs $2.6 \pm 0.2 \%, p<0.01$; Table 2 , Fig. 1$)$. This was secondary to a reduction in fenestration diameter and frequency. 
Table 1 Age, weight and blood tests for control and diabetic baboons

\begin{tabular}{lll}
\hline & $\begin{array}{l}\text { Control } \\
\text { animals }\end{array}$ & $\begin{array}{l}\text { Diabetic } \\
\text { animals }\end{array}$ \\
\hline Age (years) & $12.8 \pm 2.21$ & $12.8 \pm 2.0$ \\
Weight $(\mathrm{kg})$ & $22.3 \pm 1.2$ & $21.2 \pm 2.7$ \\
HbA $_{1 \mathrm{c}}(\%)$ & $3.6 \pm 0.25$ & $7.8 \pm 0.7^{*}$ \\
Random plasma glucose & $9.2 \pm 2.2$ & $31 \pm 6.2^{*}$ \\
$\quad$ (mmol/l) & & \\
Cholesterol (mmol/l) & $2.3 \pm 0.4$ & $2.8 \pm 0.2^{*}$ \\
Triacylglycerols $(\mathrm{mmol} / \mathrm{l})$ & $0.3 \pm 0.1$ & $2.3 \pm 0.65^{*}$ \\
Protein $(\mathrm{g} / \mathrm{l})$ & $66 \pm 4.5$ & $74 \pm 4.0^{*}$ \\
Albumin $(\mathrm{g} / \mathrm{l})$ & $34 \pm 1.7$ & $34 \pm 1.7$ \\
Bilirubin $(\mu \mathrm{mol} / \mathrm{l})$ & $3.8 \pm 1.5$ & $2.5 \pm 1.3^{*}$ \\
ALP $(\mathrm{U} / \mathrm{l})$ & $91 \pm 30$ & $297 \pm 89^{*}$ \\
GGT $(\mathrm{U} / \mathrm{l})$ & $28 \pm 3.8$ & $36 \pm 5.8^{*}$ \\
ALT $(\mathrm{U} / \mathrm{l})$ & $32 \pm 5.5$ & $52 \pm 19^{*}$ \\
AST $(\mathrm{U} / \mathrm{l})$ & $27 \pm 3.6$ & $55 \pm 48$ \\
Creatinine $(\mu \mathrm{mol} / \mathrm{l})$ & $93 \pm 2.5$ & $86 \pm 13$ \\
Haemoglobin $(\mathrm{g} / \mathrm{l})$ & $140 \pm 10$ & $129 \pm 6$ \\
White cell count $\left(\times 10^{9} / \mathrm{l}\right)$ & $7.8 \pm 3.2$ & $6.9 \pm 1.6$ \\
Platelets $\left(\times 10^{9} / 1\right)$ & $250 \pm 34$ & $350 \pm 76^{*}$ \\
\hline
\end{tabular}

Values are means \pm SEM

$A L T$ Alanine aminotransferase; $A L P$ alkaline phosphatase; $A S T$ aspartate aminotransferase; $G G T$ gamma glutamyltransferase $* p<0.05$

Transmission electron microscopy The endothelium was significantly thicker in the diabetic livers $(170 \pm 17$ vs $123 \pm$ $10 \mathrm{~nm}, p<0.01$; Table 2, Fig. 2). Stellate cells lying beneath the LSECs were noted more extensively in the diabetic livers ( $37 \pm 6$ vs $22 \pm 4 \%$ of endothelium, $p=0.04$; Fig. 3 ). There was no difference in the width of the space of Disse and extensive collagen deposition in the space of Disse was noted to similar extents in both groups.

Light microscopy and immunohistochemistry The diabetic livers were fatty with swollen hepatocytes (Figs. 3 and 4). Diabetic livers had extensive endothelial staining for von Willebrand factor, caveolin-1 and Masson's trichrome (Fig. 4). There was no difference in collagen IV staining between the two groups. PAS staining showed increased hepatocytic staining in the diabetic livers. SMA staining was increased in the diabetic compared with the control animals ( $9 \pm 12$ vs $1 \pm 1$ activated cells per field at $\times 20$ magnification, $p<0.001)$.

\section{Discussion}

The streptozotocin-induced diabetic Papio hamadryas baboon colony has proven to be a very useful animal model for the study of type 1 diabetes mellitus $[14,17,18]$. In this study, data are presented from middle-aged baboons (approximately 13 years of age, maximum life expectancy: 25-30 years) that have been treated with insulin for over a decade. Blood results revealed the expected features of diabetes mellitus such as elevated glucose, $\mathrm{HbA}_{1 \mathrm{c}}$ and markedly elevated triacylglycerols.

In this study we focussed on the effects of diabetes on the LSECs because recent reports indicate an association between pathological changes in the LSECs and dyslipidaemia [2, 4, 9]. In the diabetic livers, the LSECs were found to be about $40 \%$ thicker and the porosity of fenestrations was reduced by about $50 \%$. These are substantial changes and of a similar magnitude to those reported in old age [5-8]. In addition, diabetes was associated with increased endothelial production of von Willebrand factor and caveolin-1 and increased Masson's trichrome staining. In the normal liver, endothelial production of von Willebrand factor is minimal, but upregulation has been reported in hepatic fibrosis, cirrhosis and old age $[5,19]$. Von Willebrand factor is an endothelial glycoprotein that mediates attachment of platelets after endothelial injury; its upregulation in the LSECs is indicative of a pathophysiological state in these cells. Caveolin-1 is a protein found in fenestrations [20]. Its upregulation has been reported in cirrhosis of the liver [21]. Our finding of increased staining for the Masson's trichrome reagent is consistent with deposition of extravascular matrix. On the other hand, extensive collagen deposition was found in the space of Disse both in control and in diabetic baboons, while immunohistochemistry did not reveal any major difference in collagen IV staining in the two groups. Overall, the ultrastructural and immunohistochemical results confirm that diabetes mellitus is associated with significant changes in the hepatic sinusoid and LSECs.

Although the microvascular complications of diabetes mellitus are well established [1], few previous investigations have studied the effects of diabetes mellitus on the hepatic microvasculature or specifically studied the LSEC and its fenestrations. Berneau et al. [22] studied liver biopsies of 12 type 1 diabetic patients aged 23 to 56 years.

Table 2 Ultrastructural parameters of the LSECs in control and diabetic baboons

\begin{tabular}{|c|c|c|}
\hline & $\begin{array}{l}\text { Control } \\
\text { animals }\end{array}$ & $\begin{array}{l}\text { Diabetic } \\
\text { animals }\end{array}$ \\
\hline Porosity (\%) & $2.6 \pm 0.2$ & $1.4 \pm 0.1^{*}$ \\
\hline Fenestration diameter (nm) & $50 \pm 1$ & $43 \pm 1^{*}$ \\
\hline $\begin{array}{l}\text { Fenestration frequency (per } \\
\mu \mathrm{m}^{2} \text { ) }\end{array}$ & $12.1 \pm 0.8$ & $7.8 \pm 0.8^{*}$ \\
\hline Endothelial thickness (nm) & $123 \pm 10$ & $170 \pm 17^{*}$ \\
\hline Width of space of Disse (nm) & $754 \pm 62$ & $742 \pm 71$ \\
\hline
\end{tabular}

Values are means \pm SEM

$* p<0.01$ 
Fig. 1 Scanning electron microscope images of the luminal surface of the LSECs in diabetic baboons (a) and age-matched controls (b). There are fewer fenestrations (arrows) in the diabetic livers. Original magnification: $\times 20,000$
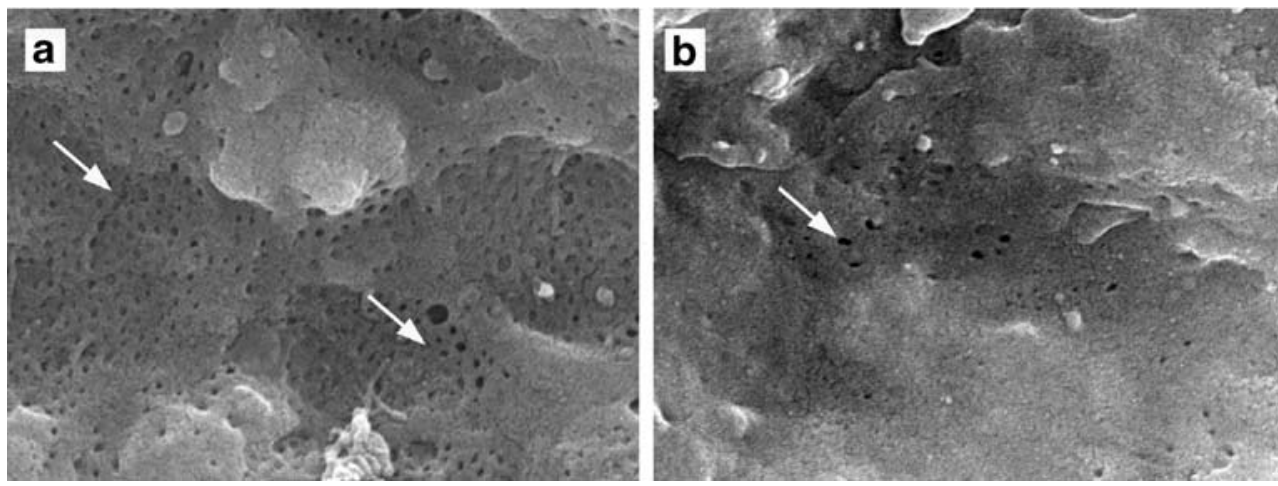

Moderate increases in collagen and basal lamina deposition in the space of Disse were reported compared with slightly younger subjects with unconjugated hyperbilirubinaemia (aged 31-42 years). Some diabetic subjects had fatty liver and an increase in endothelial Masson's trichrome staining was also noted. The same group also studied BB rats, a species in which some rats develop a condition very similar to type 1 diabetes mellitus [23]. Again, perisinusoidal deposition of collagen was noted, both in the diabetic and non-diabetic animals. In both of the above reports, the endothelial cells were stated to be unaffected. On the other hand, a pilot scanning electron microscopy study of rats 8 months after administration of streptozotocin found a small but significant increase in fenestration diameter and a reduction in fenestration frequency [24]. Latry et al. [25]
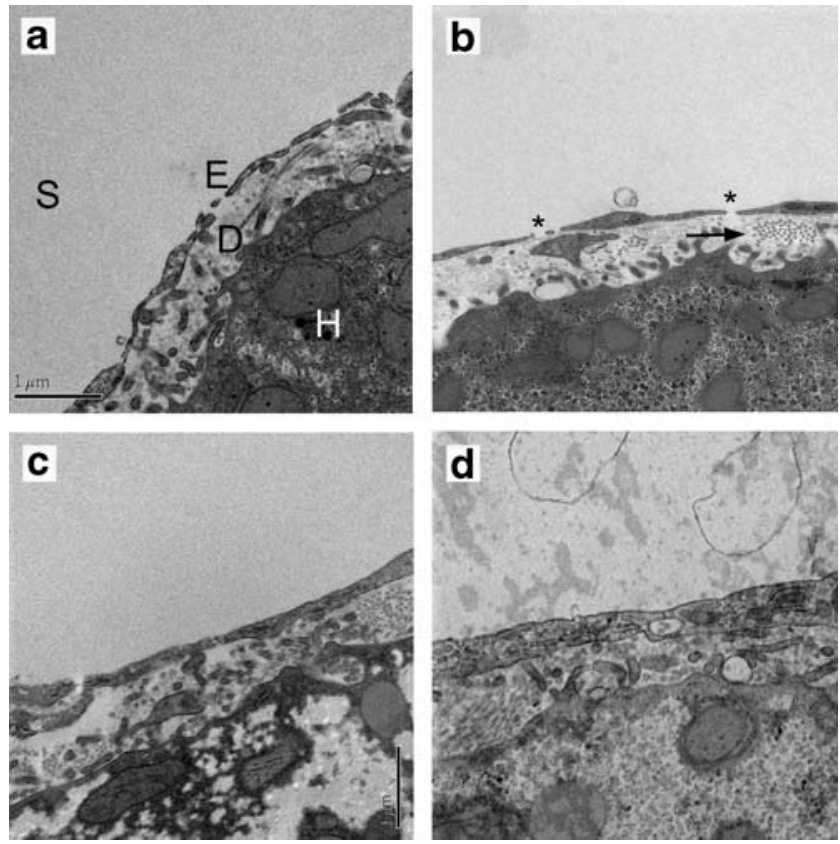

Fig. 2 Transmission electron microscope images of the LSECs in control $(\mathbf{a}, \mathbf{b})$ and diabetic $(\mathbf{c}, \mathbf{d})$ livers. In diabetic livers $(\mathbf{c}, \mathbf{d})$, the endothelium is thicker and there are less fenestrations. D, space of Disse; E, endothelium; H, hepatocyte; S, sinusoid; arrow, collagen; asterisk, fenestrations found increased deposition of collagen and basal lamina in four human diabetic liver biopsy samples. More recently, Harrison et al. [26] retrospectively studied ten type 1 and two type 2 diabetic patients who had undergone liver biopsy. Although steatohepatitis was not observed, there was extensive perisinusoidal fibrosis and deposition of basal lamina, which was termed 'diabetic hepatosclerosis'. On the whole, our results of hepatosclerosis are consistent with these previous reports; however, we also noted extensive collagen deposition in the controls, which may reflect ageing changes in the baboon [6].
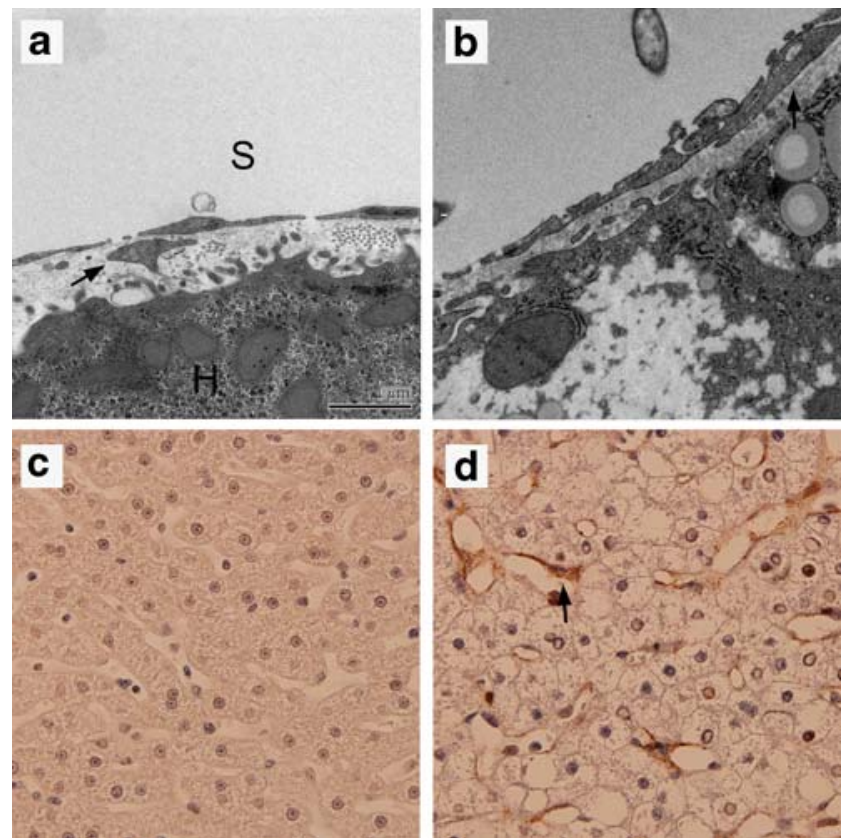

Fig. 3 Stellate cells in control $(\mathbf{a}, \mathbf{c})$ and diabetic $(\mathbf{b}$, d) livers Transmission electron microscopy shows increased stellate cells (arrows) beneath the endothelial cells in diabetic livers (b) compared with control livers (a). Sometimes fenestrations (asterisks) appeared to be blocked by stellate cells. Original magnification: $\times 20,000$. H, hepatocyte; S, sinusoid. d Immunohistochemistical staining for SMA (arrow), a marker of stellate cell activation, was extensive in the perisinusoidal regions of the diabetic livers compared with controls (c). Original magnification: $\times 60$ 

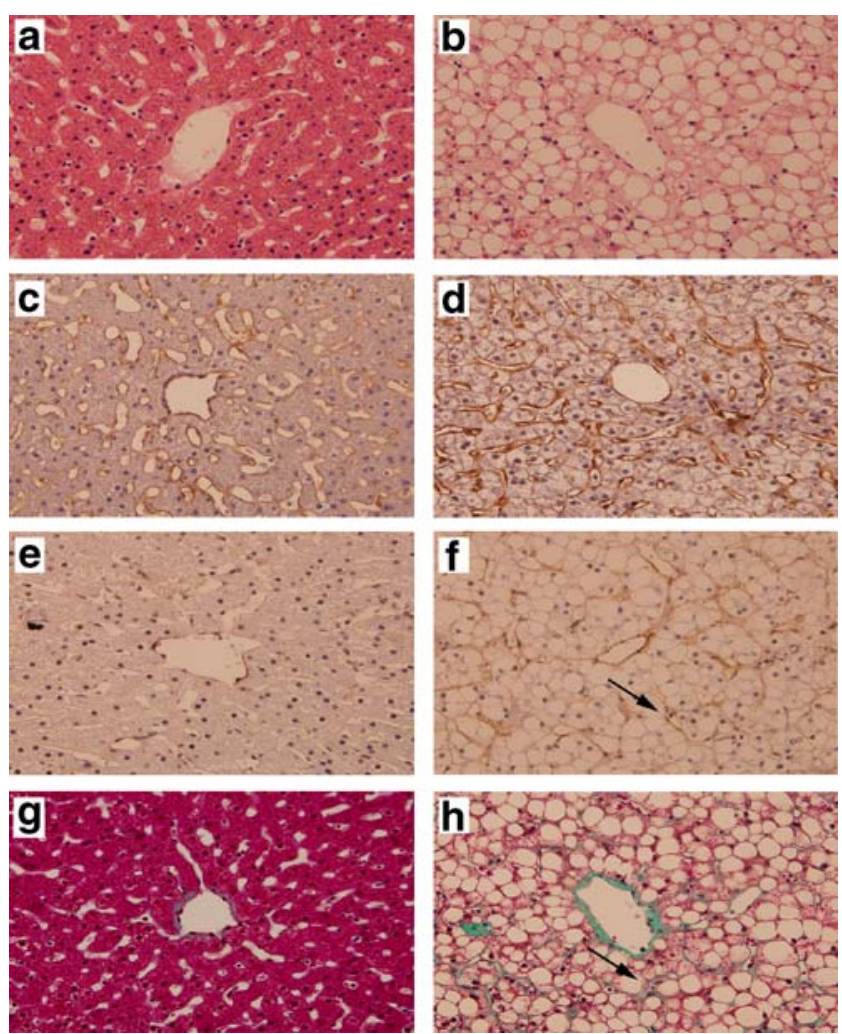

Fig. 4 Light microscopy and immunohistochemistry in control (a, c, $\mathbf{e}, \mathbf{g})$ and diabetic $(\mathbf{b}, \mathbf{d}, \mathbf{f}, \mathbf{h})$ livers. Haematoxylin and eosin $(\mathbf{a}, \mathbf{b})$, caveolin-1 (c, d), von Willebrand factor $(\mathbf{e}, \mathbf{f})$ and Masson trichrome stain $(\mathbf{g}, \mathbf{h})$. The diabetic livers showed steatosis and increased endothelial staining (arrows)

Hepatosclerosis is reported to be associated with microvascular complications of diabetes in other organ sites [26]. Consistent with this finding, our diabetic baboons have microalbuminuria and also objective changes indicative of diabetic neuropathy. While improved glycaemic control has been shown to prevent diabetes-related microvascular renal and neuropathic complications both in humans with type 1 diabetes mellitus and in animal models of diabetes $[1,12,13]$, it has yet to be determined whether hepatosclerosis can be prevented by intensive glycaemic control. Indeed, this is an important issue for future studies to address.

Here we report for the first time that diabetes mellitus is associated with defenestration and thickening of the LSECs. This has potential implications for liver function, particularly the hepatic clearance of lipoproteins such as chylomicron remnants [2]. One of the initial steps in the metabolism of lipoproteins is that chylomicron remnants pass through fenestrations into the space of Disse for receptor-mediated uptake and subsequent processing in hepatocytes. We have shown that loss of fenestrations is associated with impaired transfer of lipoproteins across the liver sinusoidal endothelium. Lipoproteins of an average diameter of $56 \mathrm{~nm}$ freely cross the liver endothelium in young rats. However, defenestration associated with old age [9] and treatment with a surfactant, poloxamer 407 [27], are associated with impaired transfer of lipoproteins and hypertriacylglycerolaemia. Delayed chylomicron remnant clearance and subsequent post-prandial hypertriacylglycerolaemia are features of type 1 diabetes [10-12]; thus it is possible that the substantial loss of fenestrations may contribute to this dyslipidaemia.

In terms of mechanisms for these changes, there was evidence of increased stellate cell numbers and activity. Fibrosis is generated by activated stellate cells and such cells are frequently noted in liver conditions such as nonalcoholic fatty liver disease and cirrhosis [28]. The diabetic group in our study also displayed increased SMA staining. Stellate cell processes were underlying a larger proportion of the endothelium in diabetic livers. In some cases, the stellate cells appeared to occlude fenestrations and potentially increase the effective diffusional distance for substrate transfer between the sinusoidal lumen and the space of Disse. Oxidative stress, which is increased in diabetes [29], also has marked effects on the structure of the LSECs $[30,31]$.

In addition, we saw mild increases in some liver function tests, while light microscopy indicated changes consistent with fatty liver. Fatty liver is very common in long-standing type 2 diabetes mellitus, particularly in older and overweight subjects [32]. The baboons in our study were nonobese and had a model of type 1 diabetes mellitus, but they were also middle-aged. Although minimal inflammation was noted, the association of steatosis with features consistent with ballooning of the hepatocytes and perisinusoidal fibrosis indicates that these diabetic baboons had type 4 non-alcoholic fatty liver disease [32]. Fatty liver is rare in type 1 diabetes mellitus and is indicative of poor glycaemic control in humans [33]. Our baboon model is a deliberate sub-optimally treated model of type 1 diabetes mellitus, with ongoing insulin deficiency and hyperglycaemia. It has features that resemble Mauriac syndrome in humans, where diabetes onset before or around the time of puberty, when under-treated, can subsequently lead to features including hepatic steatosis and reduced linear growth [34]. Hepatic steatosis in our baboon model may be caused primarily by marked insulin deficiency leading to increased lipolysis and fatty acid uptake by the liver. In turn, studies in man and animals have shown that steatosis may promote hepatic insulin resistance, thus facilitating the oxidation of fatty acids and generation of reactive oxygen species, which could lead to the production of inflammatory cytokines in hepatocytes and potentially contribute to further liver damage [32, 33, 35]. Additionally hyperglycaemia may exacerbate hepatocyte lipogenesis and steatosis in this model [35]. While other reports have 
described hepatosclerosis in the absence of demonstrable hepatic steatosis in very long standing type 1 diabetes [2426], the planned under-insulinisation in the current model probably explains why both pathologies occur in the diabetic baboons.

In conclusion, we found significant diabetes-related hepatic microvascular changes, including defenestration and thickening of the liver sinusoidal endothelium. Such changes are likely to have a significant effect on liver function and the clearance of many components of the blood, including lipoproteins. This could contribute to the pathogenesis of systemic macrovascular disease associated with diabetes mellitus.

Acknowledgements This work was supported by grants from the National Health and Medical Research Council (Australia) and the Ageing and Alzheimer's Research Foundation (Sydney, Australia). We acknowledge the support of the Pathology Department of the Concord RG Hospital, especially S. Brammah and S. Yung Kwun for assistance with the transmission electron microscopy and immunohistochemistry, respectively. We are also grateful for the electron microscopy and image analysis advice provided by T. Day (Division of Neurobiology, University of Arizona, Tucson, AZ, USA). We acknowledge the support of J. Thompson, A.-M. Hennessy, S. Thomson and S. Heffernan for the Baboon Colony, as well as the Juvenile Diabetes Research Foundation, Rebecca Cooper Foundation and National Health and Medical Research Council (Australia) for financial support of the Baboon Colony.

Duality of interest The authors declare that there is no duality of interest associated with this manuscript.

\section{References}

1. Singleton JR, Smith AG, Russell JW, Feldman EL (2003) Microvascular complications of impaired glucose tolerance. Diabetes 52:2867-2873

2. Fraser R, Dobbs BR, Rogers GW (1995) Lipoproteins and the liver sieve: the role of fenestrated sinusoidal endothelium in lipoprotein metabolism, atherosclerosis, and cirrhosis. Hepatology 21:863-874

3. Le Couteur DG, Fraser R, Hilmer S, Rivory LP, McLean AJ (2005) The hepatic sinusoid in aging and cirrhosis: effects on hepatic substrate disposition and drug clearance. Clin Pharmacokinet 44:187-200

4. Le Couteur DG, Fraser R, Cogger VC, McLean AJ (2002) Hepatic pseudocapillarisation and atherosclerosis in ageing. Lancet 359:1612-1615

5. Le Couteur DG, Cogger VC, Markus AMA et al (2001) Pseudocapillarization and associated energy limitation in the aged rat liver. Hepatology 33:537-543

6. Cogger VC, Warren A, Fraser R, Ngu M, McLean AJ, Le Couteur DG (2003) Hepatic sinusoidal pseudocapillarization with aging in the non-human primate. Exp Gerontol 38:1101-1107

7. Warren A, Bertolino P, Cogger VC, McLean AJ, Fraser R, Le Couteur DG (2005) Hepatic pseudocapillarization in aged mice. Exp Gerontol 40:807-812
8. McLean AJ, Cogger VC, Chong GC et al (2003) Age-related pseudocapillarization of the human liver. J Pathol 200:112-117

9. Hilmer SN, Cogger VC, Fraser R, McLean AJ, Sullivan D, Le Couteur DG (2005) Age-related changes in the hepatic sinusoidal endothelium impede lipoprotein transfer in the rat. Hepatology 42:1349-1354

10. Mamo JC, Elsegood CL, Umeda Y, Hirano T, Redgrave TG (1993) Effect of probucol on plasma clearance and organ uptake of chylomicrons and VLDLs in normal and diabetic rats. Arterioscler Thromb 13:231-239

11. Battula SB, Fitzsimons O, Moreno S et al (2000) Postprandial apolipoprotein B48- and B100-containing lipoproteins in type 2 diabetes: do statins have a specific effect on triglyceride metabolism? Metabolism 49:1049-1054

12. Adiels M, Olofsson SO, Taskinen MR, Boren J (2006) Diabetic dyslipidaemia. Curr Opin Lipidol 17:238-246

13. Goldberg IJ, Dansky HM (2006) Diabetic vascular disease: an experimental objective. Arterioscler Thromb Vasc Biol 26:1693-1701

14. Heffernan S, Phippard A, Sinclair A et al (1995) A baboon (Papio hamadryas) model of insulin-dependent diabetes. J Med Primatol 24:29-34

15. Rees DA, Alcolado JC (2005) Animal models of diabetes mellitus. Diabet Med 22:359-370

16. Szkudelski T (2001) The mechanism of alloxan and streptozotocin action in B cells of the rat pancreas. Physiol Res 50: $537-546$

17. Birrell AM, Heffernan SJ, Kirwan P, McLennan S, Gillin AG, Yue DK (2002) The effects of aminoguanidine on renal changes in a baboon model of type 1 diabetes. J Diabetes Complications 16: 301-309

18. Heffernan S, James V, Zilkens R et al (1996) Changes of extracellular matrix in a baboon (Papio hamadryas) model of insulin dependent diabetes: studies using electron microscopy and X-ray diffraction techniques. Diabetes Res Clin Pract 34: $65-72$

19. Hollestelle MJ, Geertzen HG, Straatsburg IH, van Gulik TM, van Mourik JA (2004) Factor VIII expression in liver disease. Thromb Haemost 91:267-275

20. Ogi M, Yokomori H, Oda $\mathrm{M}$ et al (2003) Distribution and localization of caveolin-1 in sinusoidal cells in rat liver. Med Electron Microsc 36:33-40

21. Yokomori H, Oda M, Ogi M, Sakai K, Ishii H (2002) Enhanced expression of endothelial nitric oxide synthase and caveolin- 1 in human cirrhosis. Liver 22:150-158

22. Bernuau D, Guillot R, Durand AM et al (1982) Ultrastructural aspects of the liver perisinusoidal space in diabetic patients with and without microangiopathy. Diabetes 31:1061-1067

23. Bernuau D, Guillot R, Durand-Schneider AM, Poussier P, Moreau A, Feldmann G (1985) Liver perisinusoidal fibrosis in BB rats with or without overt diabetes. Am J Pathol 120:38-45

24. Jamieson H, Dobbs BR, Day WA, Rogers GWT, Fraser R (2001) The liver sieve in diabetes: are the ultrastructural changes similar to those seen in alcoholism? In: Wisse E, Knook DL, De Zanger R, Arthur MJP (eds) Cells of the hepatic sinusoid, vol 8 . Proceedings of the 10th international symposium on cells of the hepatic sinusoid. Kupffer Cell Foundation, Leiden, pp 123-124

25. Latry P, Bioulac-Sage P, Echinard E et al (1987) Perisinusoidal fibrosis and basement membrane-like material in the livers of diabetic patients. Human Pathol 18:775-780

26. Harrison SA, Brunt EM, Goodman ZD, Di Bisceglie AM (2006) Diabetic hepatosclerosis: diabetic microangiopathy of the liver. Arch Pathol Lab Med 130:27-32

27. Cogger VC, Hilmer S, Sullivan D, Muller M, Fraser R, Le Couteur DG (2006) Surfactants and hyperlipidemia: the liver sieve is a link. Atherosclerosis 189:273-281 
28. Friedman SL (2004) Mechanisms of disease: mechanisms of hepatic fibrosis and therapeutic implications. Nat Clin Pract Gastroenterol Hepatol 1:98-105

29. Ceriello A (2006) Controlling oxidative stress as a novel molecular approach to protecting the vascular wall in diabetes. Curr Opin Lipidol 17:510-518

30. Cogger VC, Mross PE, Hosie MJ, Ansselin AD, McLean AJ, Le Couteur DG (2001) The effect of acute oxidative stress on the ultrastructure of the perfused rat liver. Pharmacol Toxicol 89:306311

31. Cogger VC, Muller M, Fraser R, McLean AJ, Khan J, Le Couteur DG (2004) The effects of oxidative stress on the liver sieve. J Hepatol 41:370-376
32. McCullough AJ (2004) The clinical features, diagnosis and natural history of nonalcoholic fatty liver disease. Clin Liver Dis 8:521-533, viii

33. Van Steenbergen W, Lanckmans S (1995) Liver disturbances in obesity and diabetes mellitus. Int J Obes Relat Metab Disord 19 (Suppl 3):S27-S36

34. Mauras N, Merimee T, Rogol AD (1991) Function of the growth hormone-insulin-like growth factor I axis in the profoundly growthretarded diabetic child: evidence for defective target organ responsiveness in the Mauriac syndrome. Metabolism 40:1106-1111

35. Riley P, O’Donohue J, Crook M (2007) A growing burden: the pathogenesis, investigation and management of non-alcoholic fatty liver disease. J Clin Pathol DOI 10.1136/jcp.2006.044891 